Pure and Applied Mathematics Quarterly

Volume 1, Number 4

(Special Issue: In Memory of

Armand Borel, Part 3 of 3 )

$817-826,2005$

\title{
Some Remarks on Gopakumar-Vafa Invariants
}

\author{
A. Schwarz and I. Shapiro
}

\begin{abstract}
We show that Gopakumar-Vafa (GV) invariants can be expressed in terms of the cohomology ring of moduli space of D-branes without reference to the $\left(\mathfrak{s l}_{2}\right)_{L} \oplus\left(\mathfrak{s l}_{2}\right)_{R}$ action. We also give a simple construction of this action.
\end{abstract}

\section{Introduction}

Our goal is to express the Gopakumar-Vafa (GV) invariants [GV] of a threedimensional Calabi-Yau manifold in terms of the cohomology ring of moduli space of D-branes. More precisely, we consider the moduli space $\mathcal{M}_{\beta}$ of holomorphic curves in a Calabi-Yau 3-fold $M$ that belong to the homology class $\beta \in H_{2}(M)$, the moduli space $\widehat{\mathcal{M}}_{\beta}$ of corresponding D-branes and the natural map $p: \widehat{\mathcal{M}}_{\beta} \rightarrow$ $\mathcal{M}_{\beta}$. (To specify a D-brane wrapping a holomorphic curve we should fix some additional data: a holomorphic line bundle or, more generally, a semi-stable coherent sheaf over the curve.)

One can construct an action of the Lie algebra $\left(\mathfrak{s l}_{2}\right)_{L} \oplus\left(\mathfrak{s l}_{2}\right)_{R}$ on the $L^{2}$ cohomology $H^{\bullet}\left(\widehat{\mathcal{M}}_{\beta}\right)$; GV-invariants are defined in terms of this action by the formula (5).

Consider an operator $L$ on $H^{\bullet}\left(\widehat{\mathcal{M}}_{\beta}\right)$ acting as multiplication by $p^{*} \omega$ where $\omega$ denotes the Kaehler class of $\mathcal{M}_{\beta}$. We will show that one can obtain an expression for GV-invariants in terms of $L$. Define $\nu_{l}^{\alpha}$ as the number of Jordan cells in the decomposition of $L$ having size $l$ and minimal degree $\alpha$.

We observe that the character of the representation of $\left(\mathfrak{s l}_{2}\right)_{L} \oplus\left(\mathfrak{s l}_{2}\right)_{R}$ on $H^{\bullet}\left(\widehat{\mathcal{M}}_{\beta}\right)$ is recovered from $\nu_{l}^{\alpha}$ as

$$
\chi(\varphi, \psi)=\sum_{l, \alpha} \nu_{l}^{\alpha} e^{i(\alpha+l-1-d) \varphi} \frac{\sin (l \psi)}{\sin \psi}
$$

Received March 3, 2005. Partly supported by NSF grant No. DMS 0204927. 
where $d=\operatorname{dim}_{\mathbb{C}} \widehat{\mathcal{M}}_{\beta}$.

To find GV-invariants $n_{r}$ one should represent the character in the form

$$
\chi(\varphi, \psi)=\sum a_{r s} 4^{r+s} \cos ^{2 r}(\varphi / 2) \cos ^{2 s}(\psi / 2)
$$

then $n_{r}=a_{r 0}$. Alternatively one can use the following explicit formula

$$
n_{r}=\sum_{\substack{l, \alpha \\ \alpha+l \geq 1+d}}(-1)^{l+1} l \nu_{l}^{\alpha}\left(c_{r}^{\alpha+l-1-d}-c_{r}^{\alpha+l-3-d}\right)
$$

where $c_{r}^{j}=(-1)^{r+j}\left(\begin{array}{c}r+j+1 \\ j-r\end{array}\right)($ see $(8))$.

The above statements can be derived from the considerations of Sec.1 and from the existence of $\left(\mathfrak{s l}_{2}\right)_{L} \oplus\left(\mathfrak{s l}_{2}\right)_{R}$ action on the cohomology of $\widehat{\mathcal{M}}_{\beta}$. The existence of such an action follows from identification of this cohomology with the space of quantum BPS states and the interpretation of BPS states in the framework of M-theory.

It was conjectured in $[\mathrm{GV}]$ that one can avoid any reference to M-theory and construct this action by means of a Leray spectral sequence associated to the map $p: \widehat{\mathcal{M}}_{\beta} \rightarrow \mathcal{M}_{\beta}$. As was shown in [HST] the classical Leray spectral sequence should be replaced by its perverse analogue (see $[\mathrm{BBD}]$ ) to give the construction of the $\left(\mathfrak{s l}_{2}\right)_{L} \oplus\left(\mathfrak{s l}_{2}\right)_{R}$ action.

In Sec.2 we discuss the construction of $\left(\mathfrak{s l}_{2}\right)_{L} \oplus\left(\mathfrak{s l}_{2}\right)_{R}$ action using the recent paper $[\mathrm{CM}]$ that allows one to give a more detailed and transparent picture than $[\mathrm{BBD}]$. We avoid using complicated mathematical notions like perverse sheaves. (We use the term "intersection cohomology", but if one believes in the so called Cheeger-Goresky-MacPherson conjecture, one can interpret intersection cohomology as $L^{2}$-cohomology up to a shift.)

In Sec.3 we give a construction of the $\left(\mathfrak{s l}_{2}\right)_{L} \oplus\left(\mathfrak{s l}_{2}\right)_{R}$ action using elementary linear algebra. The hard theorems of $[\mathrm{BBD}, \mathrm{CM}]$ can then be used to show that its character is identical to the one in [HST].

\section{Algebra of GV-invariants}

Let us consider representations of the direct sum of two copies of the Lie algebra $\mathfrak{s l}_{2}$. Irreducible representations of $\left(\mathfrak{s l}_{2}\right)_{L} \oplus\left(\mathfrak{s l}_{2}\right)_{R}$ are labelled by two non-negative integers $j_{L}, j_{R}$. To each such pair corresponds the tensor product $V_{j_{L}} \otimes V_{j_{R}}$ where $V_{j_{L}}$ and $V_{j_{R}}$ denote the irreducible representations of the left and right copy of $\mathfrak{s l}_{2}$ in $\left(\mathfrak{s l}_{2}\right)_{L} \oplus\left(\mathfrak{s l}_{2}\right)_{R}$.

The number $j$ stands for the highest weight of the $(j+1)$-dimensional representation $V_{j}$ of $\mathfrak{s l}_{2}$; physicists use the spin $s=j / 2$ to label representations. The generators of $\left(\mathfrak{s l}_{2}\right)_{L}$ and $\left(\mathfrak{s l}_{2}\right)_{R}$ will be denoted by $e_{L}, f_{L}, h_{L}$ and $e_{R}, f_{R}, h_{R}$ 
respectively; they satisfy the relations $[e, f]=h,[h, e]=2 e,[h, f]=-2 f$. Note that physicists would re-scale our $h$ by $1 / 2$.

Denote by $I$ the $\mathfrak{s l}_{2}$ representation $V_{1} \oplus V_{0} \oplus V_{0}$. Write $I_{r}$ for the $r$-th tensor power of this representation: $I_{r}=I^{\otimes r}$. One defines GV-numbers $n_{r}$ of the $\left(\mathfrak{s l}_{2}\right)_{L} \oplus\left(\mathfrak{s l}_{2}\right)_{R}$ representation $V$ in the following way. We decompose $V$ into a direct sum:

$$
V=\sum_{r} I_{r} \otimes R_{r}
$$

where $I_{r}$ is the representation of $\left(\mathfrak{s l}_{2}\right)_{L}$ defined above, and $R_{r}$ is a (virtual) representation of $\left(\mathfrak{s l}_{2}\right)_{R}$. Then $n_{r}$ is defined by the formula

$$
n_{r}=\operatorname{Tr}_{R_{r}}(-1)^{h_{R}}
$$

If $V$ is decomposed into a direct sum of irreducible representations:

$$
V=\bigoplus_{j_{L}, j_{R}} N_{j_{L}, j_{R}} V_{j_{L}} \otimes V_{j_{R}}
$$

one can write down the following simple formula for the GV-numbers:

$$
n_{r}=\sum_{j_{L}, j_{R}}(-1)^{r+j_{L}}\left(\begin{array}{c}
r+j_{L}+1 \\
j_{L}-r
\end{array}\right)(-1)^{j_{R}}\left(j_{R}+1\right) N_{j_{L}, j_{R}}
$$

This follows directly from the formula

$$
V_{j}=\sum_{r}(-1)^{r+j}\left(\begin{array}{c}
r+j+1 \\
j-r
\end{array}\right) I_{r}
$$

where the RHS is interpreted as a direct sum of virtual representations. (In other words (8) should be understood as an equality at the level of characters.)

To check (8) we write down the characters:

$$
\operatorname{char} V_{j}=\frac{\sin ((j+1) \phi)}{\sin (\phi)} \text { and } \operatorname{char} I_{r}=(2 \cos (\phi / 2))^{2 r},
$$

then use the following well known formula ( $n$ even)

$$
\frac{\sin (n a)}{\sin (a)}=\sum_{i=0}^{\infty}(-1)^{i}\left(\begin{array}{c}
n-i-1 \\
i
\end{array}\right) 2^{n-2 i-1} \cos ^{n-2 i-1}(a) .
$$

Let us consider a finite dimensional graded vector space $V=\oplus V^{n}$ equipped with an operator $L$ obeying $L V^{n} \subset V^{n+2}$. Such a structure can be specified on the space of an $\left(\mathfrak{s l}_{2}\right)_{L} \oplus\left(\mathfrak{s l}_{2}\right)_{R}$ representation $V$ by taking the grading corresponding to the diagonal Cartan operator $h_{L}+h_{R}$ and setting $L=e_{R}$. We notice that the character of the representation $V$ can be expressed in terms of this structure.

Namely, observe that $V$ can be decomposed into a direct sum of homogeneous cyclic $L$-modules, i.e. into a direct sum of subspaces spanned by vectors 
$v, L v, \cdots, L^{l-1} v$ where $v$ is homogeneous of degree $\alpha$. We call such a subspace a Jordan cell of size $l$ and minimal degree $\alpha$. Let $\nu_{l}^{\alpha}$ be the number of Jordan cells in this decomposition having size $l$ and minimal degree $\alpha$. Then the character of $V$ is recovered by the formula

$$
\operatorname{char}(V)=\sum_{l, \alpha} \nu_{l}^{\alpha} e^{i(\alpha+l-1) \varphi} \frac{\sin (l \psi)}{\sin \psi}
$$

To check the above it is sufficient to consider $V=V_{n} \otimes V_{m}$, it has $n+1$ Jordan cells of length $m+1$, with minimal degrees $-n-m,-n-m+2, \cdots, n-m$. One readily verifies the formula in this case.

Corresponding GV-invariants can be calculated by using formula (8) and the observation that $e^{-i n \varphi}+e^{i n \varphi}=\operatorname{char}\left(V_{n}\right)-\operatorname{char}\left(V_{n-2}\right)$ for $n>0$. Explicitly

$$
n_{r}=\sum_{\substack{l, \alpha \\
\alpha+l \geq 1}}(-1)^{\alpha+r} l \nu_{l}^{\alpha}\left[\left(\begin{array}{c}
\alpha+l+r \\
2 r+1
\end{array}\right)-\left(\begin{array}{c}
\alpha+l+r-2 \\
2 r+1
\end{array}\right)\right] .
$$

It is essentially the same formula as in the introduction but without the shift by $d$ caused by the inconvenience of working with the traditionally graded cohomology.

Now we make an observation to which we will return at the end of Sec.2. Suppose that the character of an $\left(\mathfrak{s l}_{2}\right)_{L} \oplus\left(\mathfrak{s l}_{2}\right)_{R}$ representation is given by $\chi(\varphi, \psi)=$ $\sum h^{p, q} e^{i p \varphi} e^{i q \psi}$, where $h^{p, q}$ are some integers. It is easy to see that the GVinvariants are then given by the formula:

$$
n_{r}=(-1)^{r} \sum_{p \geq r}(-1)^{p}\left[\left(\begin{array}{c}
p+r+1 \\
2 r+1
\end{array}\right)-\left(\begin{array}{c}
p+r-1 \\
2 r+1
\end{array}\right)\right] e\left(h^{p, \bullet}\right)
$$

where $e\left(h^{p, \bullet}\right)=\sum_{q}(-1)^{q} h^{p, q}$ is the Euler characteristic.

For practical purposes it is inconvenient and unnecessary to find a homogeneous Jordan decomposition of $V$ as described above. Instead one can obtain all pairs $(l, \alpha)$ appearing in the decomposition of $V$ via the following procedure. Pick a vector $v \in V$ of minimal degree, and let $V_{v}$ be the $L$ submodule generated by $v$. Read off $(l, \alpha)$ as the dimension of $V_{v}$ and the degree of $v$. Consider $V / V_{v}$; again a graded $L$-module. Repeat until there is nothing left.

Example. Let us consider a graded unital associative algebra $W$ generated by elements $x$ and $y$ of degree 2 obeying the relations $x^{9}=x^{8} y+x^{7} y^{2}$ and $y^{3}=0$. We define $L$ as the multiplication by $x$. Here we have to modify the discussion above to introduce the $d$ back into it.

Taking $1 \in W^{0}$ we get a pair $(10,0)$, taking $y \in W^{2}$ we get $(10,2)$, finally $y^{2} \in W^{4}$ gives $(7,4)$. From this we see that $n_{0}=27, n_{1}=-10$ and the rest are 0 . It is worthwhile to observe that we are in the situation in which we can extend the action of $L$ to that of $\left(\mathfrak{s l}_{2}\right)_{L} \oplus\left(\mathfrak{s l}_{2}\right)_{R}$ with the class of the representation being 
$V_{1} \otimes V_{9} \oplus V_{0} \oplus V_{6}$. It is easiest to obtain the GV-invariants in this way whenever possible.

This example corresponds to the case of $\mathrm{GV}$-invariants of the manifold $M$ represented as the total space of $\mathcal{O}(-3)$ over $\mathbb{C} P^{2}$ [MS]. The algebra $W$ above is isomorphic to the cohomology algebra of the space $\widehat{\mathcal{M}}_{\beta}$ where $\beta=3 \xi$ and $\xi$ is the generator of $H_{2}(M)=H_{2}\left(\mathbb{C} P^{2}\right)=\mathbb{Z}$. The element $x$ comes from the Kaehler class $\omega$ of $\mathcal{M}_{\beta}=\mathbb{C} P^{9}$, i.e. $x=p^{*} \omega$.

\section{Geometry of GV-invariants}

GV-invariants of a Calabi-Yau threefold $M$ can be defined in terms of the map $p: \widehat{\mathcal{M}} \rightarrow \mathcal{M}$ where $\mathcal{M}$ stands for the moduli space of holomorphic curves in $M$ and $\widehat{\mathcal{M}}$ denotes the moduli space of corresponding D-branes. (To specify a D-brane one needs a holomorphic curve and a (semi-stable) holomorphic vector bundle or, more generally, coherent sheaf over the curve.) It follows from physical considerations based on $M$-theory interpretation of $\widehat{\mathcal{M}}$ that $\left(\mathfrak{s l}_{2}\right)_{L} \oplus\left(\mathfrak{s l}_{2}\right)_{R}$ acts on the cohomology of $\widehat{\mathcal{M}}$. (This action can be obtained by identifying the cohomology of $\widehat{\mathcal{M}}$ with the space of quantum BPS states.) Using the action one can define $\mathrm{GV}$-invariants. More precisely, every component of $\mathcal{M}$ specifies a sequence of $\mathrm{GV}$-invariants. The components $\mathcal{M}_{\beta}$ of $\mathcal{M}$ are labelled by the homology class $\beta$ of the holomorphic curve; one can identify $\mathcal{M}_{\beta}$ with the corresponding component of the Chow variety.

It seems that the appropriate cohomology theory is $L^{2}$-cohomology; this statement is supported by the fact that in other situations it was successfully used to describe BPS states, see for example [SS]. Cheeger, Goresky and MacPherson [CGM] conjectured that for a projective variety $L^{2}$-cohomology coincides with intersection cohomology (more precisely, with intersection cohomology with the middle perversity). In this conjecture $L^{2}$-cohomology is defined by means of the standard metric on the projective space (Fubini-Study metric) restricted to the smooth part of the variety. If we accept this conjecture, it is natural to define GVinvariants in terms of the $\left(\mathfrak{s l}_{2}\right)_{L} \oplus\left(\mathfrak{s l}_{2}\right)_{R}$ action on the intersection cohomology of $\widehat{\mathcal{M}}$.

One should observe that it is not quite clear how to define rigorously the spaces $\mathcal{M}$ and $\widehat{\mathcal{M}}$ appearing in the definition of GV-invariants. (Physical considerations determine only the part of these spaces corresponding to non-singular curves.) It was suggested in [HST] to introduce $\widehat{\mathcal{M}}_{d}$ as the normalized moduli space of semistable sheaves $\mathcal{E}$ of pure dimension 1 on $M$ with Hilbert polynomial $P(\mathcal{E}, m)=$ $d \cdot m+1$. It was pointed out to us by Sheldon Katz that using the cohomology of this moduli space does not always lead to correct GV-invariants of a CalabiYau 3-fold. There exists a natural map $\widehat{\mathcal{M}}_{d} \rightarrow \mathcal{M}_{d}$ sending every sheaf $\mathcal{E}$ to its support, a curve of degree $d$, more precisely to an element of the Chow variety. (It 
is known [HL] that $\widehat{\mathcal{M}}_{d}$ is a projective scheme.) It was shown in [HST] that the $\left(\mathfrak{s l}_{2}\right)_{L} \oplus\left(\mathfrak{s l}_{2}\right)_{R}$ action on the intersection cohomology of $\widehat{\mathcal{M}}_{d}$ (more accurately, on the associated graded space of a certain filtration on $\left.I H^{\bullet}\left(\widehat{\mathcal{M}}_{d}\right)\right)$ can be obtained from the Beilinson-Bernstein-Deligne theory of perverse sheaves [BBD].

More generally, an action of $\left(\mathfrak{s l}_{2}\right)_{L} \oplus\left(\mathfrak{s l}_{2}\right)_{R}$ can be defined on the intersection cohomology $I H^{\bullet}(X)$ for any projective morphism $f: X \rightarrow Y$ and ample line bundles over $X$ and $Y$. We will discuss in more detail the $\left(\mathfrak{s l}_{2}\right)_{L} \oplus\left(\mathfrak{s l}_{2}\right)_{R}$ action on intersection cohomology $I H^{\bullet}(X)$ that corresponds to a projective map $f: X \rightarrow Y$ of projective varieties and ample line bundles $A$ over $Y$ and $\eta$ over $X$. We use $[\mathrm{CM}]$ that contains a new proof of results in $[\mathrm{BBD}]$ as well as some additional useful facts.

It will be convenient for us to use the convention for the grading of $I H^{\bullet}(X)$ that places it in degrees between $-d$ and $d$, where $d=\operatorname{dim}_{\mathbb{C}} X$. In this way the grading coincides with the weights of the Cartan operator.

The line bundles $A$ and $\eta$ determine operators $L$ and $\eta$ on the cohomology $I H^{\bullet}(X)$ defined by means of multiplication by $f^{*}(c(A))$ and $c(\eta)$ respectively where $c(A)$ and $c(\eta)$ denote the characteristic classes of $A$ and $\eta$. Note the use of $\eta$ for both the line bundle and the associated operator.

It is well known that the operator $\eta^{i}: I H^{-i}(X) \rightarrow I H^{i}(X)$ is an isomorphism. For non-singular $X$ this fact (known as the hard Lefschetz theorem) follows from standard theorems about Kaehler manifolds. Using this it is easy to construct $\mathfrak{s l}_{2}$ action on $I H^{\bullet}(X)$. One starts by finding a homogeneous basis $\left\{v_{\alpha}\right\}$ for the subspace of $I H^{\bullet}(X)$ consisting of primitive elements. $\left(v \in I H^{-i}(X)\right.$ is primitive if $\eta^{i+1} v=0$.) Let $\operatorname{deg} v_{\alpha}=-i_{\alpha}$, then $I H^{\bullet}(X)$ is a direct sum of subspaces spanned by $v_{\alpha}, \eta v_{\alpha}, \cdots, \eta^{i_{\alpha}} v_{\alpha}$. The representation of $\mathfrak{s l}_{2}$ on $I H^{\bullet}(X)$ is then defined by letting $e \in \mathfrak{s l}_{2}$ act as $\eta$, the Cartan element $h \in \mathfrak{s l}_{2}$ act as multiplication by the degree and the action of $f \in \mathfrak{s l}_{2}$ is defined inductively by requiring that $f v_{\alpha}=0$. In that way the direct sum decomposition above becomes the decomposition of $I H^{\bullet}(X)$ into irreducible $\mathfrak{s l}_{2}$ submodules.

The operator $L$ (as does every nilpotent operator) specifies a weight filtration $W_{k}$ on $I H^{\bullet}(X)$. Here it will be convenient to diverge from the convention in $[\mathrm{CM}]$ and use a decreasing filtration defined as

$$
W_{k}=\sum_{i+j=k} \operatorname{Ker} L^{1-i} \cap \operatorname{Im} L^{j} .
$$

It is characterized as the unique filtration with the properties that $L W_{i} \subset W_{i+2}$ and $L^{i}: G r_{-i} \rightarrow G r_{i}$ is an isomorphism.

Denote by $G r^{L} I H^{\bullet}(X)$ the associated graded space of the weight filtration. Since $L: I H^{\bullet}(X) \rightarrow I H^{\bullet+2}(X)$, the subspaces $W_{k}$ of $I H^{\bullet}(X)$ are homogeneous, 
i.e. $W_{k}=\bigoplus_{i} W_{k} \cap I H^{i}(X)$, and so $G r^{L} I H^{\bullet}(X)=\bigoplus G r_{i}^{L} I H^{j}(X)$; let us set $\mathcal{H}^{p, q}:=G r_{q}^{L} I H^{p+q}(X)=W_{q} \cap I H^{p+q}(X) / W_{q+1} \cap I H^{p+q}(X)$.

The operators $L$ and $\eta$ descend to $\operatorname{Gr}^{L} I H^{\bullet}(X)$. We observe that $L: \mathcal{H}^{p, q} \rightarrow$ $\mathcal{H}^{p, q+2}$ and so

$$
L^{i}: \mathcal{H}^{p,-i} \rightarrow \mathcal{H}^{p, i}
$$

is an isomorphism. (This follows directly from the characterizing properties of the weight filtration and the fact that $L$ preserves the $p$-degree.) The operator $\eta$ maps $\mathcal{H}^{p, q}$ into $\mathcal{H}^{p+2, q}$, and

$$
\eta^{i}: \mathcal{H}^{-i, q} \rightarrow \mathcal{H}^{i, q}
$$

is an isomorphism. This is a consequence of the identification of $\mathcal{H}^{p, q}$ with (following the notation of $[\mathrm{CM}]$ ) the so called graded perverse cohomology groups $H_{-p}^{d+p+q}$, where $d=\operatorname{dim}_{\mathbb{C}} X$. This identification (in other words, coincidence of the weight filtration of $L$ with the perverse filtration associated with the map $f$ ) is one of the main results of $[\mathrm{CM}]$. Similarly one can say that $\mathcal{H}^{p, q}$ is identified with the $E_{2}^{q p}$ term of the perverse spectral sequence associated to the map $f$. (It is the same as the $E_{\infty}^{q p}$ term.) Now the isomorphism (16) is the so called relative hard Lefschetz theorem of [BBD, CM].

Following an identical procedure to the one used in the construction of the $\mathfrak{s l}_{2}$ action on $I H^{\bullet}(X)$, we can use isomorphisms (15), (16) to obtain an $\left(\mathfrak{s l}_{2}\right)_{L} \oplus\left(\mathfrak{s l}_{2}\right)_{R}$ action on $G r^{L} I H^{\bullet}(X)$. In particular $e_{L}$ and $e_{R}$ act as $\eta$ and $L$ respectively and the Cartan operators $h_{L}$ and $h_{R}$ multiply $\mathcal{H}^{p, q}$ by $p$ and $q$. If $v \in \mathcal{H}^{-p,-q}$ is a primitive element (i.e. $L^{q+1} v=\eta^{p+1} v=0$ ) then the elements $L^{i} \eta^{j} v, 0 \leq i \leq q$, $0 \leq j \leq p$ span an irreducible $\left(\mathfrak{s l}_{2}\right)_{L} \oplus\left(\mathfrak{s l}_{2}\right)_{R^{-}}$invariant subspace of $G_{r}{ }^{L} I H^{\bullet}(X)$. The lifting of the $\left(\mathfrak{s l}_{2}\right)_{L} \oplus\left(\mathfrak{s l}_{2}\right)_{R}$ action to $I H^{\bullet}(X)$ will be discussed later.

Example. Let us consider the case of $f: \widehat{M}_{X} \rightarrow M$, where $M$ is a smooth projective variety, $X$ a smooth closed subvariety, and $\widehat{M}_{X}$ is the blowup of $M$ along $X$. Denote by $d$ the codimension of $X$ in $M$. As usual, let $L$ denote the operator of multiplication by the pullback along $f$ of the Chern class of an ample line bundle on $M$. Then as an $L$-module $H^{\bullet}\left(\widehat{M}_{X}\right)=H^{\bullet}(M) \oplus H^{\bullet}(X)[-2] \oplus \cdots \oplus$ $H^{\bullet}(X)[-2(d-1)]$, where $L$ acts on the summands in an obvious way.

Thus it follows from the preceding discussion that the GV-invariants are $n_{0}=$ $(-1)^{\operatorname{dim}\left(\widehat{M}_{X}\right)} e\left(\widehat{M}_{X}\right)=(-1)^{\operatorname{dim}(M)}(e(M)+(d-1) e(X))$ and for $r>0, n_{r}=$ $(-1)^{\operatorname{dim}(M)+r}\left(\begin{array}{c}d+r-1 \\ 2 r+1\end{array}\right) e(X)$. The above formulas are most conveniently obtained by observing that as an $\left(\mathfrak{s l}_{2}\right)_{L} \oplus\left(\mathfrak{s l}_{2}\right)_{R}$-module $G r^{L} H^{\bullet}\left(\widehat{M}_{X}\right)=V_{0} \otimes H^{\bullet}(M) \oplus$ $V_{d-2} \otimes H^{\bullet}(X)$. 
To reconcile the weight filtration approach with the discussion of homogeneous Jordan cells in Sec.1 we observe that we may define the weight filtration associated to the operator $L$ acting on $V$ as follows. We decompose $V$ into a direct sum of cyclic $L$-modules spanned by $v, L v, \cdots, L^{l} v$ and define a grading on $V$ by placing $v$ in degree $-l$ and setting the degree of $L$ to be 2 . This grading is admittedly non-canonical however the associated filtration $W_{k}=\bigoplus_{i \geq k} V^{i}$ satisfies the characterizing property of the weight filtration of $L$ and so is equal to it. Furthermore this grading gives an $L$-equivariant isomorphism $V \rightarrow G r^{L} V$ of graded spaces.

This is readily modified to the case when $V$ was already graded and $L$ had degree 2 with respect to this grading by considering the homogeneous Jordan cell decomposition. Here we get a bi-graded $V$ and an $L$-equivariant isomorphism $V^{\bullet \bullet} \rightarrow G r_{\bullet}^{L} V^{\bullet}$ of bi-graded spaces. As a consequence of the above we see that $I H^{\bullet}(X)$ itself has a non-canonical bi-grading with total grading coinciding with the usual one, and so there is an $L$-equivariant isomorphism $\operatorname{IH}(X)^{\bullet \bullet} \rightarrow \mathcal{H}^{\bullet \bullet}$ of bi-graded spaces. Unfortunately this isomorphism is non $\eta$-equivariant, and so the $\left(\mathfrak{s l}_{2}\right)_{L} \oplus\left(\mathfrak{s l}_{2}\right)_{R}$ action is strictly speaking constructed naturally only on $G r^{L} I H^{\bullet}(X)$, however it can be lifted to $I H^{\bullet}(X)$ via the above isomorphism. In any case, if one is willing to believe that $\left(\mathfrak{s l}_{2}\right)_{L} \oplus\left(\mathfrak{s l}_{2}\right)_{R}$ acts on $I H^{\bullet}(X)$ in such a way that the grading corresponds to the action of the diagonal Cartan operator $h_{L}+h_{R}$ and that $e_{R}$ acts by $L$, then the character of this representation will be the same as the one obtained from the action of $\left(\mathfrak{s l}_{2}\right)_{L} \oplus\left(\mathfrak{s l}_{2}\right)_{R}$ on $\operatorname{Gr}^{L} I H^{\bullet}(X)$ discussed here.

In light of the above discussion we see that the character of the $\left(\mathfrak{s l}_{2}\right)_{L} \oplus\left(\mathfrak{s l}_{2}\right)_{R}$ action on $G r^{L} I H^{\bullet}(X)$ can be written down in terms of $\mathcal{H}^{p, q}$. More precisely, let $h^{p, q}=\operatorname{dim} \mathcal{H}^{p, q}$, then $\chi(\varphi, \psi)=\sum h^{p, q} e^{i p \varphi} e^{i q \psi}$, and the GV-invariants are given by the formula (13). We can interpret $e\left(h^{p, \bullet}\right)$ as the Euler characteristic of ${ }^{\text {perv }} R^{p} f_{*} I C_{X}$, a perverse sheaf on $Y$.

\section{A simple definition of the $\left(\mathfrak{s l}_{2}\right)_{L} \oplus\left(\mathfrak{s l}_{2}\right)_{R}$ action}

We begin by making the following general observation. Let $A$ and $B$ be any two commuting nilpotent operators on a vector space $V$. Denote by $W_{k}$ the weight filtration of $V$ associated to $B$, then $A W_{k} \subset W_{k}$ and so $A$ and $B$ descend to $G r^{B} V$, the associated graded space. Furthermore, let $W_{k}^{i}$ be the weight filtration of $G r_{i}^{B} V$ associated to $A$. Recall that $B^{i}: G r_{-i}^{B} V \rightarrow G r_{i}^{B} V$ is an isomorphism and now $B^{i} W_{k}^{-i} \subset W_{k}^{i}$ so that

$$
B^{i}: G r_{k}^{A} G r_{-i}^{B} V \rightarrow G r_{k}^{A} G r_{i}^{B} V
$$

is an isomorphism. For any $k$ we also have that

$$
A^{i}: G r_{-i}^{A} G r_{k}^{B} V \rightarrow G r_{i}^{A} G r_{k}^{B} V
$$


is an isomorphism. As a formal consequence of (17) and (18) (refer to Sec.2) we see that $G r^{A} G r^{B} V$ has a canonical structure of an $\left(\mathfrak{s l}_{2}\right)_{L} \oplus\left(\mathfrak{s l}_{2}\right)_{R}$-module with $e_{L}=A, e_{R}=B ; h_{L}$ and $h_{R}$ act on $G r_{p}^{A} G r_{q}^{B} V$ by $p$ and $q$ respectively.

In our particular situation let $V=I H(X)$ (the • is missing to indicate that we forgot the grading), $A=\eta$ and $B=L$. We then get an $\left(\mathfrak{s l}_{2}\right)_{L} \oplus\left(\mathfrak{s l}_{2}\right)_{R}$ action on $\operatorname{Gr}^{\eta} G r^{L} I H(X)$ and so may define GV-invariants of this action. It is an immediate consequence of the results in $[\mathrm{CM}]$ that the character of the representation above is the same as in [HST], and consequently so are the GVinvariants. (More precisely, $\operatorname{dim} G r_{p}^{\eta} G r_{q}^{L} I H(X)=\operatorname{dim} \mathcal{H}^{p, q}=\operatorname{dim} E_{2}^{q p}$, where $E_{2}^{q p}$ refers to the perverse spectral sequence associated to the map $f$.) Note that if we reverse the order of the operators and consider $G r^{L} G r^{\eta} I H^{\bullet}(X)$, we do not get anything new, i.e. we canonically obtain $I H^{\bullet}(X)$ with the $\left(\mathfrak{s l}_{2}\right)_{L}$ acting trivially and $\left(\mathfrak{s l}_{2}\right)_{R}$ acting via the hard Lefschetz.

\section{Remarks}

i) In the construction of the $\left(\mathfrak{s l}_{2}\right)_{L} \oplus\left(\mathfrak{s l}_{2}\right)_{R}$ action we have used operators $L$ and $\eta$, however the character of the representation is determined by the bi-grading coming from the perverse filtration on the $I H^{\bullet}(X)$ associated to the projective map $f: X \rightarrow Y$ and so depends only on the map. GV-invariants are defined in terms of the character of the representation and so depend only on $f$ as well.

Let us consider a connected projective family of maps $f_{s}: X_{s} \rightarrow Y_{s}$ of projective varieties labelled by a parameter $s \in S$. Then the corresponding $G V$ invariants (and, more generally the character of the $\left(\mathfrak{s l}_{2}\right)_{L} \oplus\left(\mathfrak{s l}_{2}\right)_{R}$ representation) do not depend on $s \in S$ if the cohomology of $X_{s}$ does not vary over $S$. More precisely, given maps of projective varieties $\mathcal{X} \stackrel{F}{\rightarrow} \mathcal{Y} \stackrel{\alpha}{\rightarrow} S$, for every $s \in S$ we can consider maps $X_{s} \stackrel{f_{s}}{\longrightarrow} Y_{s}$ where $Y_{s}=\alpha^{-1}(s), X_{s}=F^{-1}\left(Y_{s}\right)$ and $f_{s}$ is the restriction of $F$ to $X_{s}$. Let us assume that $S$ is connected and for every $s \in S$ there is a neighborhood $U \subset S$ containing $s$ with the property that the inclusion of $X_{s}$ into $X_{U}=(\alpha \circ F)^{-1}(U)$ induces an isomorphism of the intersection cohomology. Then the $\left(\mathfrak{s l}_{2}\right)_{L} \oplus\left(\mathfrak{s l}_{2}\right)_{R}$ representations corresponding to the morphisms $f_{s}: X_{s} \rightarrow Y_{s}$ are (non-canonically isomorphic) for all $s \in S$.

ii) In the important paper $[\mathrm{KKV}], \mathrm{GV}$-invariants of a Calabi-Yau 3-fold $X$ were expressed in terms of the Euler characteristics $e\left(C^{(\delta)}\right)$, where $C^{(\delta)}$ is roughly speaking the moduli space of holomorphic curves of degree $d$ in $X$, together with a choice of $\delta$ points. (In $[\mathrm{KKV}]$ it is assumed that these curves have generic genus g.) It is interesting to note that our formula (13) is equivalent to Conjecture 3 (as it appears in $[\mathrm{K}]$ ) if we are able to verify the equality

$$
e\left(\mathcal{H}^{g-\delta, \bullet}\right)=(-1)^{\operatorname{dim} M+\delta}\left(e\left(C^{(\delta)}\right)-2 e\left(C^{(\delta-1)}\right)+e\left(C^{(\delta-2)}\right)\right)
$$


or its inverse

$$
e\left(C^{(\delta)}\right)=(-1)^{\delta+\operatorname{dim} M} \sum_{i=0}^{\delta}(-1)^{i}(i+1) e\left(\mathcal{H}^{g-\delta+i, \bullet}\right),
$$

where $e\left(\mathcal{H}^{p, \bullet}\right)=\sum_{q}(-1)^{q} \operatorname{dim} \mathcal{H}^{p, q}\left(\right.$ with $\mathcal{H}^{p, q}$ defined using the map $f: \widehat{\mathcal{M}}_{d} \rightarrow$ $\mathcal{M}_{d}$ ) and can be interpreted as the Euler characteristic of the perverse sheaf ${ }^{p e r v} R^{p} f_{*} I C_{\widehat{\mathcal{M}}_{d}}$ on $\mathcal{M}_{d}$.

Acknowledgements. We are indebted to J. Bernstein, D. Fuchs, S. Gukov, M. Kapranov, S. Katz and A. Polishchuk for interesting discussions.

\section{REFERENCES}

[BBD] A. Beilinson, J. Bernstein, P. Deligne, Faisceaux pervers, Asterisque, 100 (1982).

[CGM] J. Cheeger, M. Goresky, R. MacPherson, $L^{2}$-cohomology and intersection homology of singular algebraic varieties, Seminar on Differential Geometry, pp. 303-340, Ann. of Math. Stud., 102, Princeton Univ. Press, Princeton, N.J., 1982.

[CM] M. A. A. de Cataldo, L. Migliorini, The Hodge theory of algebraic maps, Preprint math.AG/0306030 v2.

[GV] R. Gopakumar, C. Vafa, M-Theory and Topological Strings-II, Preprint hep-th/9812127.

[HL] D. Huybrechts, M. Lehn, The Geometry of Moduli Spaces of Sheaves, Aspects of Mathematics, Vol E31, Vieweg.

[HST] S. Hosono, M.-H. Saito, A. Takahashi, Relative Lefschetz action and BPS state counting, Preprint math.AG/0105148 v2.

[K] S. Katz, Gromov-Witten, Gopakumar-Vafa, and Donaldson-Thomas invariants of CalabiYau threefolds, Preprint math.AG/0408266.

[KKV] S. Katz, A. Klemm, C. Vafa, M-Theory, Topological Strings and Spinning Black Holes, Preprint hep-th/9910181.

[MS] K. Hori et al., Mirror Symmetry, Clay Math. Monographs, Vol. 1.

[SS] G. Segal, A. Selby, The cohomology of the space of magnetic monopoles, Comm. Math. Phys., 177 (1996), no.3 775-787

\section{A. Schwarz}

Department of Mathematics, University of California, Davis, CA, USA

E-mail address: schwarz@math.ucdavis.edu

I. Shapiro

Department of Mathematics, University of California, Davis, CA, USA

E-mail address: ishapiro@math.ucdavis.edu 